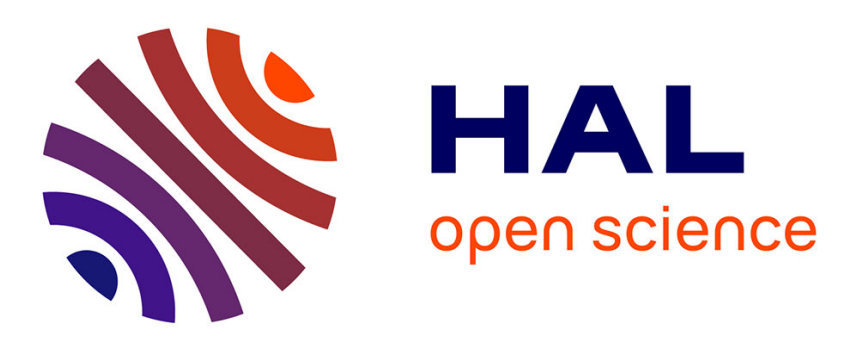

\title{
Insights into the selection mechanism of Widmanstätten growth by phase-field calculations
}

Hocine Lebbad, Benoît Appolaire, Yann Le Bouar, Alphonse Finel

\section{To cite this version:}

Hocine Lebbad, Benoît Appolaire, Yann Le Bouar, Alphonse Finel. Insights into the selection mechanism of Widmanstätten growth by phase-field calculations. Acta Materialia, 2021, 217, pp.117148. 10.1016/j.actamat.2021.117148 . hal-03333692

\section{HAL Id: hal-03333692 \\ https://hal.science/hal-03333692}

Submitted on 3 Sep 2021

HAL is a multi-disciplinary open access archive for the deposit and dissemination of scientific research documents, whether they are published or not. The documents may come from teaching and research institutions in France or abroad, or from public or private research centers.
L'archive ouverte pluridisciplinaire $\mathbf{H A L}$, est destinée au dépôt et à la diffusion de documents scientifiques de niveau recherche, publiés ou non, émanant des établissements d'enseignement et de recherche français ou étrangers, des laboratoires publics ou privés. 


\title{
Insights into the selection mechanism of Widmanstätten growth by phase-field calculations
}

\author{
H. Lebbad ${ }^{\mathrm{a}}$, B. Appolaire ${ }^{\mathrm{b}, \mathrm{c}, *}$, Y. Le Bouar ${ }^{\mathrm{a}}$, A. Finel ${ }^{\mathrm{a}}$ \\ ${ }^{a}$ Université Paris-Saclay, ONERA, CNRS, LEM, Châtillon, France \\ ${ }^{b}$ Université de Lorraine, CNRS, IJL, Nancy, France \\ ${ }^{c}$ Labex Damas, Université de Lorraine, Nancy, France
}

\begin{abstract}
We investigate the growth of Widmanstätten structures in metallic alloys with phase field calculations. We show that elastic energy anisotropy associated with shear dominated transformations is alone sufficient to give rise to plate or needle like precipitates with constant lengthening rates at constant supersaturations. We show that Ivantsov analytical solutions still provide good estimates of the diffusion flux around tips even when elasticity is accounted for. A careful analysis demonstrates that, for shear dominated transformations, (i) the lengthening direction lies in the habit plane determined by one of the minima of the elastic kernel; (ii) the tip shape and size are equilibrium features, driven by elastic forces, rather than dynamically determined as usually assumed; (iii) the tip size of acicular precipitates can be rationalized by the value of the elastic kernel in the lengthening direction.
\end{abstract}

Keywords: phase transformation, phase field, elasticity, Widmanstätten

\section{Introduction}

Since they have been discovered at the beginning of the $19^{\text {th }}$ century in meteoritic Fe-Ni [1, 2, 3, 4], Widmanstätten structures have been observed in many metallic alloys, such as Fe-C [5], Al-Ag [6], Ag-Cd [7], Cu-Zn [8, 9] and Ti alloys [10, 11], among others. These microstructures, ensuing from diffusioncontrolled phase transformations, display acicular morphologies (plates or needles), often growing from grain boundaries of the parent phase in colonies of precipitates with parallel habit planes. They are observed at intermediate temperatures or after coolings at moderate rates. In isothermal conditions, the precipitates lengthen at constant rate.

Understanding the formation of these microstructures has long been relying on either the prediction of the lenghtening kinetics or the description of the interface structure. Along the first line, all works start with the analysis of the diffusion problem around self-similar acicular shapes growing at constant velocity, following the pioneer works of $[12,13,14,15,16]$. However, the resulting models have faced two major issues. First, the diffusion problem is not closed and must be supplied with a selection criterion to obtain unique tip velocity and radius. Second, discrepancies were noted between the predictions and the few available measurements in different alloys, e.g. [17]. Starting in the $60 \mathrm{~s}$, Aaronson and co-workers were convinced that the above descriptions were not sufficient because they do not account for the interface structure that should play an active role in the growth process, explaining both its kinetics and the resulting morphology. Using TEM observations and bicrystallography, they identified interfacial ledges as the main vec-

${ }^{*}$ Corresponding author tor of interface propagation. Based on these works, competing models were proposed that assume that the propagation is controlled by the diffusion of solute species at the ledge risers $[18,19,20]$. Unfortunately, these models did not improve the agreement with the experiments, e.g. [21]. The discussion between these two routes did not end on some real conclusion, but rather on a standstill. In fact, it can be noted that all these works have neglected the role of elastic energy associated with the lattices mismatch, although regularly mentioned as the source of discrepancies, and sometimes partially accounted for [22, 23]. Using the capabilities of phase field models coupled with elasticity and plasticity [24, 25, 26, 27, 28, 29], Cottura et al. [30] have shown recently that the general features of Widmanstätten structures can be recovered by simply accounting for the anisotropic elastic energy produced by the change in crystal structure. Indeed, they have shown that there is a direction of constant lengthening rate provided that the elastic energy displays a particular dependency with respect to the interface orientation (see also [31] for a discussion on its relation to the invariant line), that seems to be a feature common to the different alloys where Widmanstätten structures are observed. However, although they have clearly shown that the eigenstrain carries all the necessary information for understanding the direction of growth, the selection mechanism of the tip size has remained puzzling. This is the issue that we address numerically in the present work.

After a brief explanation of the phase field model that we have used, a careful characterization of the steady plate lengthening in different conditions will be carried out so as to be critically compared to analytical solutions and available measurements. Using this analysis, a selection criterion for the tip size and velocity will be proposed. 


\section{Phase-field model}

The model used in the present work is the same as in [30]. It relies on a phase-field parameter $\varphi(x, t)$ that distinguishes the matrix $(\varphi=0)$, the precipitate $(\varphi=1)$ and the interface $(0<$ $\varphi<1)$. The microstructure is also described by concentration $c(\boldsymbol{x}, t)$ and displacement $u(\boldsymbol{x}, t)$ fields.

The mesoscopic free energy $\tilde{F}$ of the system is split into three contributions.

$$
\tilde{F}=W d^{3} F=W d^{3} \int_{V} d V\left[f_{c h}(\varphi, c)+f_{\text {int }}(\varphi)+f_{e l}(\boldsymbol{u}, \varphi)\right]
$$

where $W$ and $d$ are respectively the energy density and space scales and where all quantities in the integral (and so $F$ ) are non-dimensional.

The chemical contribution accounts for the free energy approximated in each phase by a parabola with respect to $c$, then interpolated within the interface,

$$
f_{c h}(c, \varphi)=\frac{K_{c}}{2}[c-h(\varphi)]^{2}
$$

where $h(\varphi)=3 \varphi^{2}-2 \varphi^{3}$ and where it is assumed, without loss of generality, that the equilibrium concentrations are 0 in the matrix and 1 in the precipitate. For simplicity, we have also considered that the curvature $K_{c}$ of the free energies with respect to concentration are the same for both phases.

The interface contribution reads:

$$
f_{\text {int }}(\varphi)=\varphi^{2}(1-\varphi)^{2}+\frac{\lambda}{2}|\nabla \varphi|^{2}
$$

with $\nabla$ the non-dimensional gradient operator, scaled by $d$. The elastic contribution reads:

$$
f_{e l}(\boldsymbol{u}, \varphi)=\frac{1}{2} \varepsilon_{i j}^{e}(\boldsymbol{u}, \varphi): C_{i j k l}: \varepsilon_{k l}^{e}(\boldsymbol{u}, \varphi)
$$

where $C$ is the fourth order tensor of elastic constants scaled by $W$, supposed to be homogeneous for simplicity. In the small strain framework, the elastic strain is given by $\varepsilon_{i j}^{e}=$ $\varepsilon_{i j}-\varepsilon_{i j}^{*}(\varphi)=\frac{1}{2}\left(\nabla_{i} u_{j}+\nabla_{j} u_{i}\right)-\varepsilon_{i j}^{*}(\varphi)$. Considering the matrix as the reference, the eigenstrain writes $\varepsilon_{i j}^{*}=h(\varphi) \varepsilon_{i j}^{T}$ where $\varepsilon_{i j}^{T}$ characterizes the change of crystal structure in the precipitate with respect to the matrix.

To ensure that the free energy functional decreases with time, relaxation dynamics are assumed for both $c(\mathbf{x}, t)$ and $\varphi(\mathbf{x}, t)$. The time evolutions of these fields read:

$$
\begin{aligned}
& \frac{\partial \varphi}{\partial t}=-L \frac{\delta F}{\delta \varphi} \\
& \frac{\partial c}{\partial t}=\frac{1}{K_{c}} \nabla^{2} \frac{\delta F}{\delta c}
\end{aligned}
$$

where the time scale is defined as $\tau=d^{2} / D$ with $D$ the interdiffusion coefficient, and where the phase field mobility $L$ is a positive constant.
Because the relaxation of displacements $u(\mathbf{x}, t)$ is much faster than diffusion, static mechanical equilibrium is considered:

$$
\nabla_{j} \cdot \frac{\delta F}{\delta \varepsilon_{i j}}=0
$$

All equations are expressed in Fourier space to transform space differential operators into algebraic operations. Time is discretized using a semi-implicit first order Euler scheme. Considering for simplicity homogeneous elastic constants $C_{i j k l}$, Eq. (7) can be solved very efficiently using the Fourier transform of the normalized Green function for displacements whose inverse is given by $\Omega_{i j}^{-1}(\boldsymbol{n})=C_{i k l j} n_{k} n_{l}$ where $\boldsymbol{n}=\boldsymbol{k} / k$ is a unit vector of reciprocal space. Moreover, we can take advantage of Fourier space to express the elastic energy as:

$$
E=\frac{1}{2} f \frac{d^{3} k}{(2 \pi)^{3}} B(\boldsymbol{n})|\theta(\boldsymbol{k})|^{2}
$$

where the $f$ is the Cauchy principal value of the integral, and where $\theta(\varphi(\boldsymbol{x}, \boldsymbol{t}))$ describes the microstructure $(\theta(\varphi(\boldsymbol{x}, \boldsymbol{t})=0)=0$ and $\theta(\varphi(\boldsymbol{x}, \boldsymbol{t})=1)=1)$.

$$
B(\boldsymbol{n})=\varepsilon_{i j}^{*} C_{i j k l} \varepsilon_{k l}^{*}-n_{i} \sigma_{i j}^{*} \Omega_{j k}(\boldsymbol{n}) \sigma_{k l}^{*} n_{l}
$$

is the elastic kernel, with $\sigma_{i j}^{*}=C_{i j k l} \varepsilon_{k l}^{*}$. The analysis of our results will strongly rely on the anisotropy of the elastic energy density that can be visualized simply with polar plots of this kernel.

The interface energy $\sigma_{\text {int }}$ and width $\delta$ are related to the energy density $W$ and length scale $d$ by $\sigma_{\text {int }}=W d \sqrt{\lambda} /(3 \sqrt{2})$ and $\delta=2 d \sqrt{2 \lambda}$, where $\lambda$ is the non-dimensional gradient coefficient. We have selected $\lambda=1.92$. This choice ensures that the interface width $\delta$ is large enough with respect to the discretization length $d$ used for the numerical implementation of the gradient operator and Fourier transforms. With the present choice, the interfaces are resolved with 5 to 6 nodes. Moreover, we have selected $K_{c}=0.77$.

Typical values for the interface energy and width can be obtained by selecting appropriate values for the energy density scale $W$ and length scale $d$. For example, $d=0.8 \mathrm{~nm}$ and $W=7.65 \times 10^{8} \mathrm{~J} \mathrm{~m}^{-3}$, interface energy is $\sigma_{i n t}=200 \mathrm{~mJ} \mathrm{~m}^{-1}$ and interface width is $\delta=3.2 \mathrm{~nm}$. Then, apart from section 4 , the elastic tensor is assumed isotropic with Lamé coefficients $\lambda_{0}=200 \mathrm{GPa}$ and $\mu_{0}=50 \mathrm{GPa}$. Finally, to ensure that the overall dynamics is controlled by diffusion, the phase field mobility $L$ must be sufficiently larger than one. We have selected $L=6.49$.

\section{Growth dynamics of 2D plates and 3D needles}

2D calculations have been performed considering $5120 \times 512$ grids. Initially, circular nuclei with radius $20 d$ are inserted at the center of the boxes, and the matrix is supersaturated with concentrations $c_{\infty}$ in the range $[0.1 ; 0.35]$. According to Cottura et al. [30], the simplest eigenstrain giving rise to Widmanstätten 
structures writes, in the frame of the computation box ([001] being perpendicular to the $2 \mathrm{D}$ section of interest):

$$
\varepsilon_{2 \mathrm{D}}^{*}=\varepsilon_{0}\left(\begin{array}{ccc}
0 & 0 & 0 \\
0 & -1 & 0 \\
0 & 0 & 0
\end{array}\right)
$$

with $\varepsilon_{0}$ the amplitude of the eigenstrain, ranging from $1 \%$ to $5 \%$. Indeed, this eigenstrain has been shown to generate an elastic energy highly anisotropic with respect to the interface orientation: the 2D polar plot of the corresponding elastic kernel $B(\boldsymbol{n})$ displays two opposite maxima along [010] (noted $\boldsymbol{e}_{3}$ ) and two opposite minima along [100] (noted $\left.\boldsymbol{e}_{1}\right)$ corresponding to the habit plane normals (Fig. 1a) such as to make a figure eight. The analytical expression of $B(\boldsymbol{n})$ is given in Appendix A.

$3 \mathrm{D}$ calculations have been performed considering $128 \times 128 \times$ 512 grids. Spherical nuclei with radius $20 d$ are inserted at the center of the boxes and initial concentrations in the matrix are set so that the supersaturations range from 0.2 to 0.35 . As shown by Cottura et al. [30], the following eigenstrain in the frame of the computation box:

$$
\varepsilon_{3 \mathrm{D}}^{*}=\varepsilon_{0}\left(\begin{array}{ccc}
0 & -1 & 0 \\
-1 & 0 & 0 \\
0 & 0 & 0
\end{array}\right)
$$

is typical of real systems displaying Widmanstätten structures (e.g. steels, Ti alloys or brass). The 3D polar plot corresponding elastic kernel is shown in Fig. 1b. It features two pairs of minima along direction [100], noted $\boldsymbol{e}_{1}$, and direction [010], noted $\boldsymbol{e}_{2}$, and one pair of maxima along direction [001], noted $\boldsymbol{e}_{3}$ : as for the $2 \mathrm{D}$ case, the minima are associated with possible habit planes. In the simple case of $\varepsilon_{3 \mathrm{D}}^{*}$ given in (11), the minima and maximum directions are mutually orthogonal. It is worth noting that the $\left(\boldsymbol{e}_{i \neq 3}, \boldsymbol{e}_{3}\right)$ sections of the 3D polar plot are similar to the $2 \mathrm{D}$ polar plot of $\varepsilon_{2 \mathrm{D}}^{*}$, such that a cylindrical nucleus with axis along either $\boldsymbol{e}_{1}$ or $\boldsymbol{e}_{2}$ would give plates similar to the $2 \mathrm{D}$ calculations. However, considering quadratic calculation boxes with equivalent sides along $\boldsymbol{e}_{1}$ and $\boldsymbol{e}_{2}$ together with an isotropic nucleus and periodic boundary conditions will give rise to Widmanstätten needles rather than plates.

In $2 \mathrm{D}$, the nuclei grow rapidly as plates with the longest direction along $\boldsymbol{e}_{3}$ (Fig. 2). In $3 \mathrm{D}$, due to the particular boundary conditions, the spherical nuclei grow rapidly as needles with the longest direction also along $\boldsymbol{e}_{3}$ (Fig. 3). In both cases, the lengthening rate is constant (after a short transient stage) at a given supersaturation and can easily be obtained by a linear fit of length vs time.

In $2 \mathrm{D}$, we have measured the radius of curvature of the tips following the protocol described in [30]. In 3D, we have followed [32] as illustrated in Fig. 3. In both cases, the measurement uncertainty is lower than a grid spacing $d$. The overall 3D shape of the tip (corresponding to $\varphi=0.5$ ) is shown together with sections perpendicular to the lengthening direction $\boldsymbol{e}_{3}$. The projections of these sections in the $\left(\boldsymbol{e}_{1}, \boldsymbol{e}_{2}\right)$ plane are plotted in 4. The needles display planar sides oriented normal to $\boldsymbol{e}_{1}$ and (a)
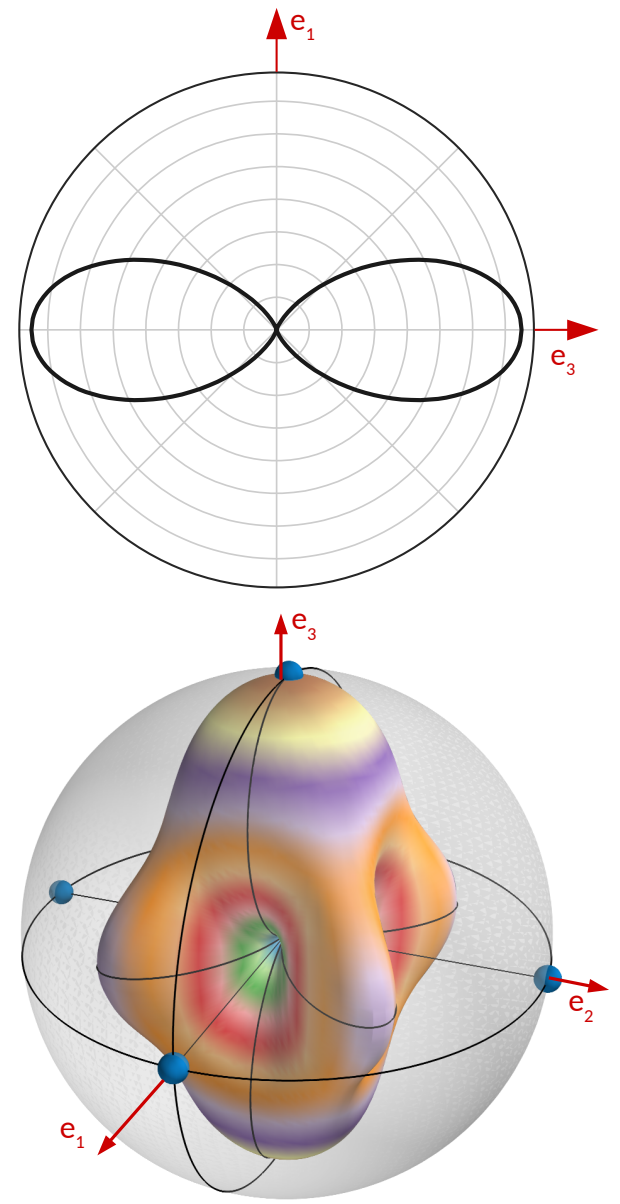

(b)

Figure 1: Polar plots of the elastic kernel with eigenstrain given by (a) $\varepsilon_{2 \mathrm{D}}^{*}$ and (b) $\varepsilon_{3 \mathrm{D}}^{*}$

$\boldsymbol{e}_{2}$ and an axisymmetric tip along $\boldsymbol{e}_{3}$. The area of the precipitate section (defined by $\varphi>0.5$ ) is plotted in Fig. 4 as a function of the distance from the very tip: the slope $\alpha$ of the linear fit gives the radius of curvature $\sqrt{\alpha / \pi}$ of the corresponding paraboloid of revolution.

The lengthening velocity $V$ and tip radius $R$ are used to compute the Péclet number $P=R V /(2 D) . \quad P$ is plotted in Fig. 5 (full symbols) with respect to the tip supersaturation defined as $\Omega=c_{\infty}-c_{i}$ where $c_{i}$ is the interfacial concentration on the matrix side at the tip. This concentration is obtained by extrapolating the concentration profile in the matrix (where $\phi<0.05$ ) close to the diffuse interface along the lengthening axis up to $\phi=0.5$.

We have also plotted the predictions of Ivantsov model [12] for the growth of plates and needle in infinite supersaturated matrix. For a parabolic plate (2D), $P$ is implicitly related to the supersaturation as:

$$
\Omega=\operatorname{Iv}_{2 \mathrm{D}}(P)=\sqrt{\pi P} \exp (P) \operatorname{erfc}(\sqrt{P})
$$

For a paraboloidal needle (3D), the implicit relation reads:

$$
\Omega=\operatorname{Iv}_{3 \mathrm{D}}(P)=P \exp (P) \mathrm{E}_{1}(P)
$$




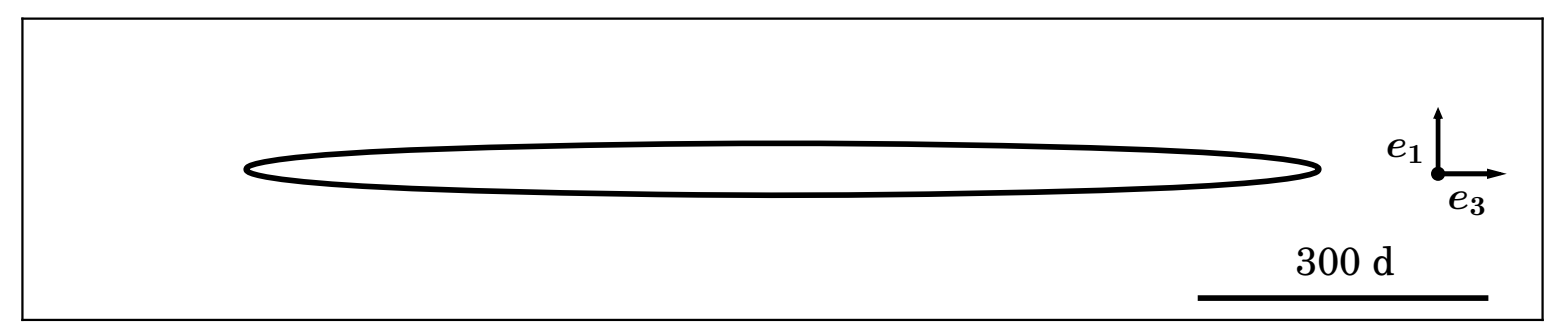

Figure 2: Level set $\varphi=0.5$ during growth (last recorded time) for $\varepsilon_{0}=4 \%$ and $\Omega=0.15$.

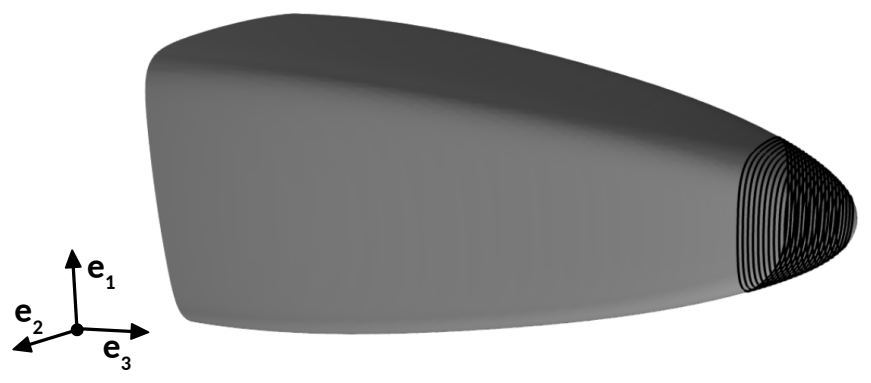

Figure 3: Needle tip given by level set $\varphi=0.5\left(\Omega=0.30\right.$ and $\varepsilon_{0}=3 \%$ at $t=9 \mathrm{~s})$. Intersections by transverse planes plotted at $z \in[1 ; 19]$.

where $\mathrm{E}_{1}$ is the exponential integral. It must be stressed that theses analytical solutions assume local equilibrium at the interface without any influence of curvature or elasticity, i.e. interfacial concentrations are provided by the phase diagram.

Overall, the phase field calculations (full symbols) are close to Ivantsov solutions (continuous lines). For 2D plates, all phase field outcomes (red symbols) are slightly above the Ivantsov red curve, whatever the supersaturations and eigenstrain amplitudes. For a given eigenstrain, the scaling of $P$ with $\Omega$ is close to Ivantsov scaling $\left(P \propto \Omega^{2.5}\right.$ in the range of supersaturations investigated, slightly above the scaling $P \propto \Omega^{2}$ corresponding to the lowest order expansion of $\operatorname{Iv}_{2 \mathrm{D}}$ when $P \rightarrow 0$ ). For 3D needles, the phase field outcomes (black symbols) are slightly more scattered with respect to the Ivantsov black curve than in 2D, with values above Ivantsov for 4 and $5 \%$ eigenstrains, and below for $3 \%$ eigenstrain. In this last case, the scaling of $P$ with $\Omega$ seems to be larger than Ivantsov. These differences may have several origins.

(i) First, the periodic boundary conditions are likely to influence the tip velocity. Indeed, although the $2 \mathrm{D}$ periodic box has been selected by successively increasing its dimensions until converging to the same results, this process has not been fully possible in 3D due to the associated computational costs (in terms of CPU and memory). Still, the ratios between the diffusion lengths $D / V$ to the lateral sizes of the boxes are only of the order of unity for the smallest supersaturations in both 2D and 3D (see supplemental material). Consequently, this may not be the main reason for explaining the differences with Ivantsov

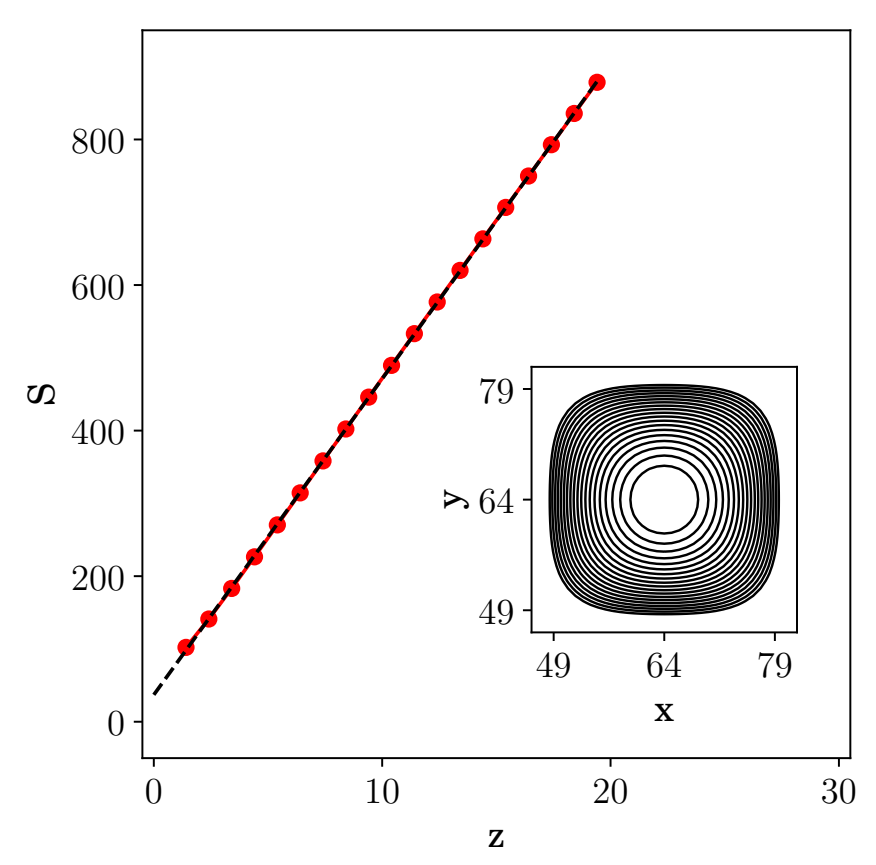

Figure 4: Transverse section area $S$ of the needle vs. the distance $z$ from the tip, determined from the projections of $\varphi=0.5$ onto $\left(\boldsymbol{e}_{1}, \boldsymbol{e}_{2}\right)$ plane (inset).

solutions.

(ii) Second, the shapes are not strictly parabolic or paraboloidal. This is pronounced for 3D with flat sides not far from the tip (Fig. 3). For 2D, we have quantitatively compared the tip shapes with parabolae, as illustrated in Fig. 6: the tips are sharper than parabolae with equivalent tip curvatures (blue circle), with stronger deviations (i.e. earlier along the long axis) from the parabolae for larger eigenstrain amplitudes. It must be emphasized that the shapes do not depend on the growth condition, i.e. supersaturation $c_{\infty}$, as illustrated in Fig. 6: this is an important outcome of our calculations that will be elaborated further in $\S 4$.

(iii) Finally, the interface is not a concentration level set, as clearly shown in Fig. 7. This observation has been overlooked in [30] where only concentration level sets far from the tips were plotted. Although it is difficult to 


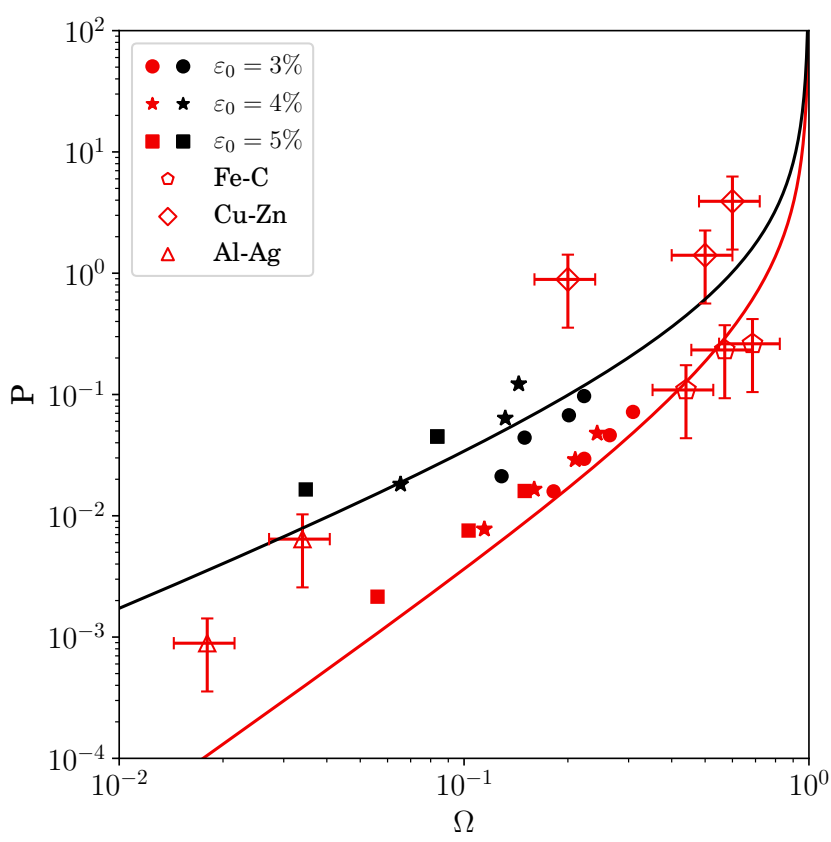

Figure 5: Péclet number $P$ vs tip supersaturation $\Omega$ : plates (2D) in red and needles (3D) in black. Ivantsov solutions are plotted with lines, phase field calculations with solid symbols, and experimental data with empty symbols (see also Tab. 1).

prove that local equilibrium holds at the interface, it can be shown that Gibbs-Thomson together with elasticity should play the major role to set the interfacial concentrations (see supplemental material).

To conclude this comparison, the predicted deviations from Ivantsov solutions can be considered as moderate in view of the significant eigenstrain investigated. Additional calculations performed with more complex eigenstrains (see \$4) predict a similar proximity to Ivantsov solutions, provided that the lengthening direction be associated with the maximum of elastic energy, as discussed in [33]. This explains why simplified models based on Ivantsov solutions are very often successful when the tip radius is tuned (e.g. [11,34]).

The validity of Ivantsov solutions for describing Widmanstätten growth has often been questioned due to the disagreements observed with some experiments. Hence, we have compared our results with respect to available experiments to see whether a better agreement is found. For that purpose, we have first collected the measurements available in the literature. Then, we have selected those where all necessary parameters can really be obtained, in particular where tip radii are provided (Tab 1). The three resulting sets of values are plotted with open symbols in Fig. 5: ferrite (bcc) plates in austenite (fcc) in Fe$\mathrm{C}$ [35] with pentagons; $\alpha$ (fcc) needles grown from $\beta$ (bcc) in $\mathrm{Cu}-\mathrm{Zn}[8,9]$ with diamonds; and $\gamma$ (hcp, also noted as $\delta$ or $\zeta$ in different phase diagrams) plates growth from $\alpha$ (fcc) in $\mathrm{Al}-\mathrm{Ag}$ [6] with triangles. We have intentionally discarded some measurements previously compared to Ivantsov [21]: (i) those concerning alloyed steels $[34,36]$ because the analysis should ac- count for the diffusion of all species and possibly for the different conditions at the interface (local equilibrium vs. paraequilibrium); (ii) those related to the dissolution of Widmanstätten microstructure in Al-15\% Ag alloy [37] because dissolution is not simply reverse growth. Unfortunately, we had also to discard a number of interesting investigations where the tip radius have been obtained indirectly from the measured kinetics using some modified Ivantsov solution [15] and the maximum growth criterion (e.g. [7]).

It seems that the experimental data are not in better agreement with our calculations than with Ivantsov solution, even if they concern different ranges of supersaturations. It can be noted that accounting arbitrarily for only $20 \%$ of uncertainty of Péclet number and supersaturation moderates the disagreements (Fig. 5). We believe that this uncertainty can be much higher owing to the difficulty to retrieve dynamical 3D features from 2D observations (most often post-mortem), as well as to estimate accurately supersaturations and diffusivities. In that respect, a few comments on the data in Tab. 1 are worth to be done.

In [35], velocities and radii have been obtained from hundred of measurements with an accuracy of about $5 \mathrm{~nm}$ so that they can be considered as quite reliable (although Fig. 2 and Tab. III related to the measurements are not consistent in this article). The supersaturations have been estimated using the $\mathrm{Fe}-\mathrm{C}$ phase diagram provided in [35], accounting for the Gibbs-Thomson effect. It must be stressed that this correction improves significantly the agreement with Ivantsov. Moreover, in view of the large supersaturations in the investigated $\mathrm{Fe}-\mathrm{C}$ alloys, interactions between neighboring plates could be a reason why the data are below the Ivantsov curve. Finally, as already suggested in [35], carbon diffusivity is averaged over the concentration range in austenite. For that purpose, we have used a more recent estimate by [39].

For the needles in $\mathrm{Cu}-\mathrm{Zn}$ brass, the values provided in [8] at $400{ }^{\circ} \mathrm{C}$ and in [9] at $520^{\circ} \mathrm{C}$ have been obtained from 20 measurements following a distribution surprisingly flat (Tab. I in [8]). Moreover, the radii of the thinnest needles could not be measured and they have been attributed the resolution limit of $50 \mathrm{~nm}$. It is not clear whether the supersaturations have been corrected to account for the curvature. Finally, the assessment of the interdiffusion coefficient entering $P$ from the tracer diffusion determined by [40] depends on the thermodynamic description of $\mathrm{Cu}-\mathrm{Zn} \beta$ phase. We have used the thermodynamic factor proposed in [8] although no detail is given on how it has been obtained.

For $\mathrm{Al}-15 \% \mathrm{Ag}$, the tip velocities have been determined from in situ observations using hot stage transmission electron microscopy. Although not mentioned, it is likely that the number of measurements is not large due to the difficulty to perform such experiments. The radius of curvature could not be measured directly and it has been assumed to be equal to the measured plate thickness. In case where the tips are very sharp, such an assumption can overestimate the radii by one order of magnitude. Moreover, besides the uncertainty associated with the determination of the supersaturation and diffusivity already noted for the other alloys, it must be stressed that the plates fea- 


\begin{tabular}{|cccccccc|}
\hline Alloy & Ref. & $\begin{array}{c}\text { Temperature } \\
\left({ }^{\circ} \mathrm{C}\right)\end{array}$ & Supersaturation & $\begin{array}{c}\text { Growth velocity } \\
\left(\mathrm{nm} \mathrm{s}^{-1}\right)\end{array}$ & $\begin{array}{c}\text { Tip radius } \\
(\mathrm{nm})\end{array}$ & $\begin{array}{c}\text { Diffusivity } \\
\left(\times 10^{-14} \mathrm{~m}^{2} \mathrm{~s}^{-1}\right)\end{array}$ & $\begin{array}{c}\text { Symbol } \\
\text { in Fig. } 5\end{array}$ \\
\hline $\mathrm{Al}-15$ wt.\%Ag & {$[6]$} & 400 & 0.034 & 6.50 to 7.35 & 20 & $1.08[38]$ & $\Delta$ \\
& & 425 & 0.018 & 1.41 to 2.62 & 20 & $2.27[38]$ & $\Delta$ \\
\hline $\mathrm{Cu}-44.1$ at.\%Zn & {$[8]$} & 400 & 0.2 & 160 & 130 to 250 & 1.8 & $\diamond$ \\
$\mathrm{Cu}-41.1$ at.\%Zn & {$[8]$} & 400 & 0.6 & $4.7 \times 10^{3}$ & $<50$ & 1.8 & $\diamond$ \\
& {$[9]$} & 520 & 0.5 & $30 \times 10^{3}$ & $100-200$ & 160 & $\diamond$ \\
\hline $\mathrm{Fe}-0.24$ wt.\%C & & & 0.686 & $21 \times 10^{3}$ & 9.5 & $38.3[39]$ & $\checkmark$ \\
$\mathrm{Fe}-0.33$ wt.\%C & {$[35]$} & 700 & 0.570 & $13 \times 10^{3}$ & 15.5 & $43.3[39]$ & $\checkmark$ \\
$\mathrm{Fe}-0.43$ wt.\%C & & & 0.440 & $6 \times 10^{3}$ & 18 & $49.6[39]$ & $\checkmark$ \\
\hline
\end{tabular}

Table 1: Growth conditions and features inferred from in situ and post mortem measurements of Widmanstätten structures in different binary alloys.

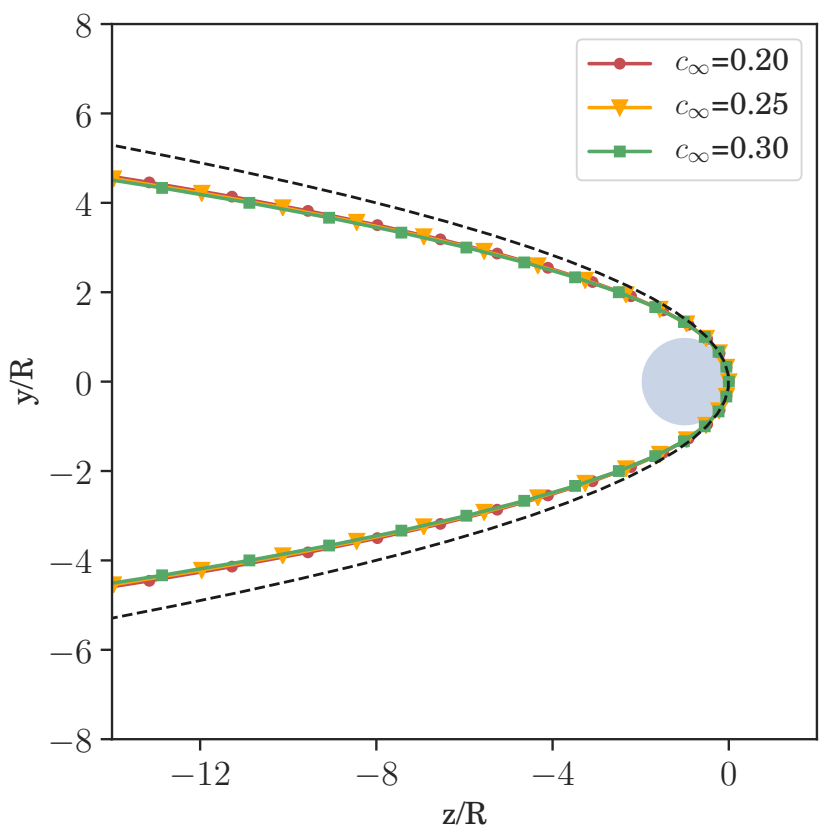

Figure 6: Tip shapes rescaled with the tip radius $R$ (defined by the blue circle) during steady-state growth with $\varepsilon_{0}=4 \%$ for different supersaturations. Dashed line: parabola with unit curvature radius.

ture very anisotropic shapes. Finally, it is also likely that growth in thin foils is modified with respect to growth in bulk.

Hence, although there is no doubt that the experimental data are invaluable, it must be recognized that they are excessively difficult to obtain and that their processing require many careful steps. Considering their insufficient accuracy, it seems they cannot be used to draw definite conclusion concerning their agreement with the Ivantsov predictions.

\section{Tip shape and size}

Two important outcomes of the previous section are:

- the tip shapes seem to be independent of the growth conditions (Fig. 6);

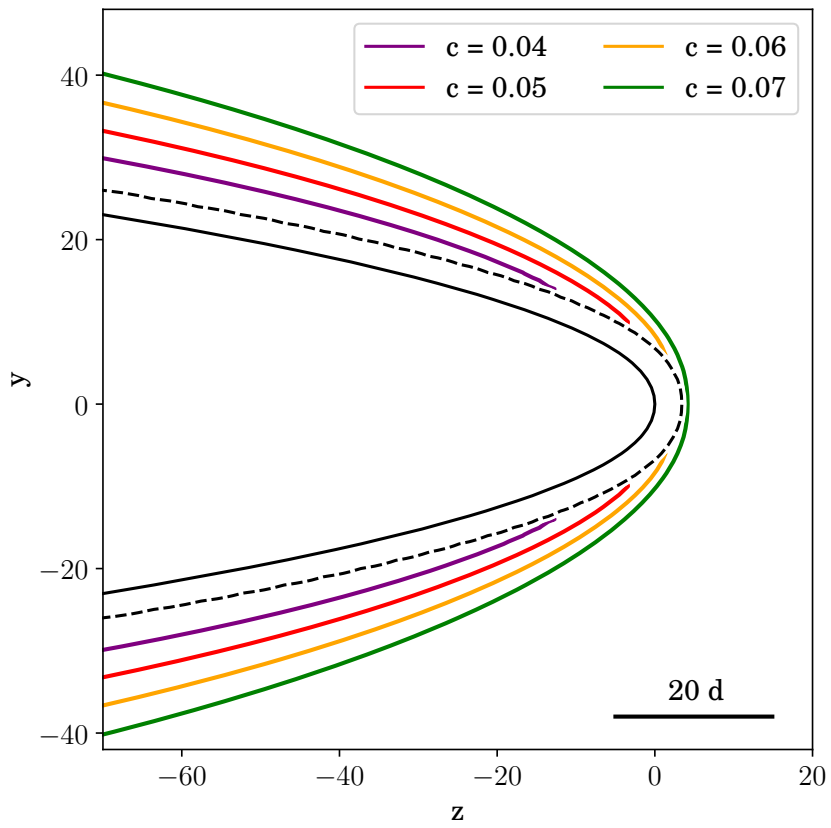

Figure 7: Concentration level sets in matrix close to interface (continuous and dashed black lines indicate level sets $\varphi=0.5$ and $\varphi=0.05$ respectively) during steady-state growth with $\varepsilon_{0}=3 \%$ and $\Omega=0.3$. 
- they are not parabolic or paraboloidal (Fig. 6) as usually assumed in analytical modeling of Widmanstätten plates.

Both statements are consequences of the role of elasticity anisotropy that can be examined with polar plots of the elastic kernel $B(\boldsymbol{n})$.

To check that our results are not specific to the very simple eigenstrains in Eqs. (10) and (11), we have considered in this section the eigenstrain related to the transformation from bec $\beta$ phase to hcp $\alpha$ phase in Ti or $\mathrm{Zr}$ and their alloys. This eigenstrain can be expressed theoretically following the Burgers mechanism [41] and using the lattice parameters of both phases. Shi et al. [42] have proposed the following one:

$$
\varepsilon_{\mathrm{Ti}}^{*}=10^{-2}\left(\begin{array}{ccc}
-8.3 & -0.9 & 0 \\
-0.9 & 12.3 & 0 \\
0 & 0 & 3.5
\end{array}\right)
$$

based on room temperature lattice parameters and accounting for interfacial misfit dislocations observed by TEM. It describes a shear dominated transformation like Eqs. (10) and (11), but it is also associated with some volume variation. It is worth noting that different shear amplitudes can be found depending on the way the lattice parameters are obtained. For example, an eigenstrain closer to Eq. (11) with $\varepsilon_{0}=10 \%$ is obtained using lattice parameters measured at high temperature by synchrotron Xray diffraction in [43, 31, 33]. Hence, working with Eq. (14) will emphasize the generic nature of the results obtained with the simple eigenstrains.

Our aim is to demonstrate a correlation between the tip shape and the elastic kernel $B(\boldsymbol{n})$ (Eq. (9)). To do so, we have modified the shape of the elastic kernel by artificially changing the values of the elastic constants. For convenience, we have used a cubic elastic tensor in the reference frame used in (14), and we have controlled the shape of the elastic kernel $B(\boldsymbol{n})$ by modifying the anisotropy ratio $A=2 C_{44} /\left(C_{11}-C_{12}\right)$. More precisely, the three components $C_{11}, C_{12}$ and $C_{44}$ have been determined so as to give the same bulk modulus $K=1 / 3\left(C_{11}+2 C_{12}\right)=55$ and the same apparent isotropic shear modulus $G\left(C_{11}, C_{12}, C_{44}\right)=13$, as defined by Kröner homogenization scheme [44, 45]. The shape change of the elastic kernel for the investigated parameter range $(A \in[1 ; 5])$ is presented in Fig. 8. $B(\boldsymbol{n})$ always displays two pairs of minima. However, the overall shape of the elastic kernel is significantly modified between $A=1$ (Fig. 8a) and $A=5$ (Fig. 8b). Note that we observed numerically that the directions of the minima do not do not change when varying the value of $A$ in the considered range.

To relate the elastic kernel to the tip shape, we have performed 2D simulations. The relevant plane for the $2 \mathrm{D}$ simulations, where growth is able to reach a stationary stage, is selected considering the polar plot of $B(\boldsymbol{n})$ as follows[30]. We first define $\boldsymbol{e}_{1}$ as the direction of one pair of minima. $\boldsymbol{e}_{2}$ is the vector normal to $\boldsymbol{e}_{1}$ in the plane containing the two pairs of minima (as shown in Fig. 8, the pairs of minima are not normal to each others contrary to Fig. 1)). Then $\boldsymbol{e}_{3}$ is defined as $\boldsymbol{e}_{3}=\boldsymbol{e}_{1} \times \boldsymbol{e}_{2}$ and lies in the habit plane by construction. The 2D simulation is then performed in the plane normal to $\boldsymbol{e}_{2}$. The relevant elastic kernel for the 2D simulations is the cut of the 3D kernel in the $\left(\boldsymbol{e}_{1}, \boldsymbol{e}_{3}\right)$ plane ( black circles in Fig. 8).

These sections of the elastic kernels in plane $\left(\boldsymbol{e}_{1}, \boldsymbol{e}_{3}\right)$ are plotted for $A=1,3$ and 5 in Fig. 9 to highlight the change in the maxima directions when increasing $A$ beyond a threshold value close to 4 . For low $A$, the maxima are aligned with direction $\boldsymbol{e}_{3}$ and $B(\boldsymbol{n})$ decreases monotonously from $\boldsymbol{e}_{3}$ to $\boldsymbol{e}_{1}$. For $A=5$, the maxima are at $45^{\circ}$ with respect to $\boldsymbol{e}_{3}$ (and $\boldsymbol{e}_{1}$ ) such that the variation of $B(\boldsymbol{n})$ is not monotonous between $\boldsymbol{e}_{3}$ and $\boldsymbol{e}_{1}$.

We now investigate how the shape of the elastic kernel impacts the tip shape and kinetics using 2D phase field simulations with different values of $A$ between 1 and 5. As expected, for symmetry reasons, the lengthening always proceeds along the horizontal direction, i.e. along $\boldsymbol{e}_{3}$. The corresponding tip shapes during growth with $c_{\infty}=0.25$ are plotted in Fig. 10 . Whereas for $A=1$ the tip is still acicular as in the previous section, the difference is striking for $A=5$ with a blunted tip with a nearly flat side perpendicular to the lengthening direction. This comparison clearly shows that the tip shape is related to how the elastic kernel behaves far from the habit plane direction $\boldsymbol{e}_{1}$ and cannot be predicted by the usual expansion of $B(\boldsymbol{n})$ around $\boldsymbol{e}_{1}$ (e.g. $\$ 8.1 .2$ and $§ 8.1 .3$ in [46]). Despite the lack of close analytical form to predict the shape, the present calculations provide some numerical evidence that acicular tip shapes are obtained when the lengthening direction $\boldsymbol{e}_{3}$, perpendicular to the habit plane normal $\boldsymbol{e}_{1}$ by symmetry, corresponds to the maximum of the elastic kernel in plane $\left(\boldsymbol{e}_{3}, \boldsymbol{e}_{1}\right)$.

As mentioned at the beginning of the section, the tip size does not seem to depend on the growth condition for a given eigenstrain (e.g. Fig. 6). This is in contrast with dendritic growth during solidification [47, 48] as in other diffusion-controlled growth problems where the selection is not ensured by the diffusion process [49]. Indeed, the calculations of $\$ 3$ have shown that the larger $\varepsilon_{0}$ the thinner the plates, with tip radii independent of the undercooling in the investigated range. It appears that this trend is not specific to the simple eigenstrains $\varepsilon_{2 D}^{*}$ or $\varepsilon_{3 D}^{*}$, provided that there exists a plane where the elastic kernel features a figure eight with only one maximal direction. This shape is likely to be obtained for any shear dominated transformation with moderate elastic constant anisotropy, as illustrated above. As can be inferred from Fig. 10, the tip size seems to be related to the maximum value $B_{\max }$ of the elastic kernel in plane $\left(\boldsymbol{e}_{1}, \boldsymbol{e}_{3}\right)$ : the largest the maximum, the sharpest the tip. In order to substantiate this statement, we have performed calculations considering two different elastic kernels displaying figure eight ( $A=1$ and $A=2$ ) with the same maximum, as shown in Fig. 11. To keep the same maximum, the isotropic bulk and shear moduli were rescaled. We have measured the steady state tip radii in both cases: they are equal within the precision of the measurement (their difference is about $8 \%$ of $d$ ). Hence, $B_{\max }$ is the most important quantity to rationalize the tip radius selection, at least for figure eight $B(\boldsymbol{n})$. Qualitatively, it is not surprising that the tip size results from the competition between the interface energy and some volumic elastic energy at the tip related to $B_{\max }$.

We have gathered the results for plates (2D) and needles 


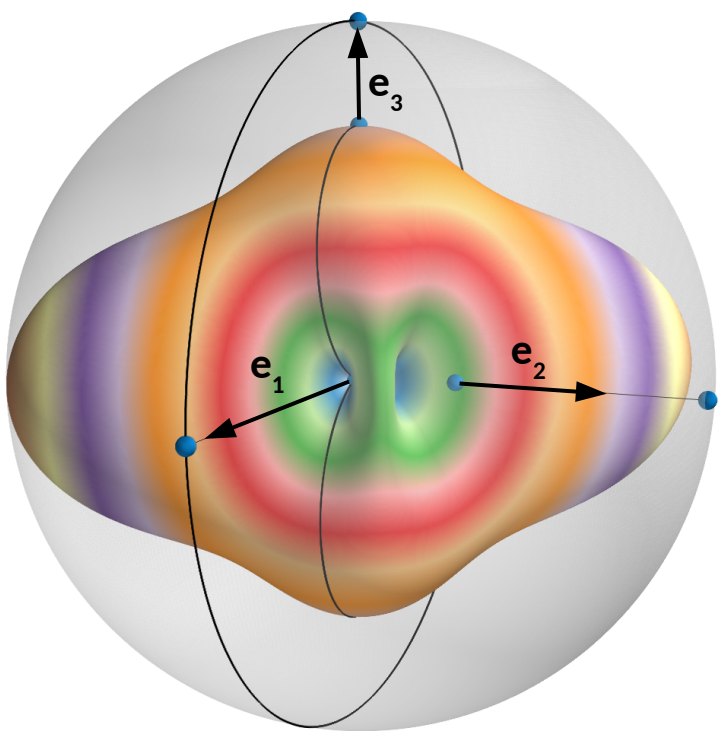

(a)

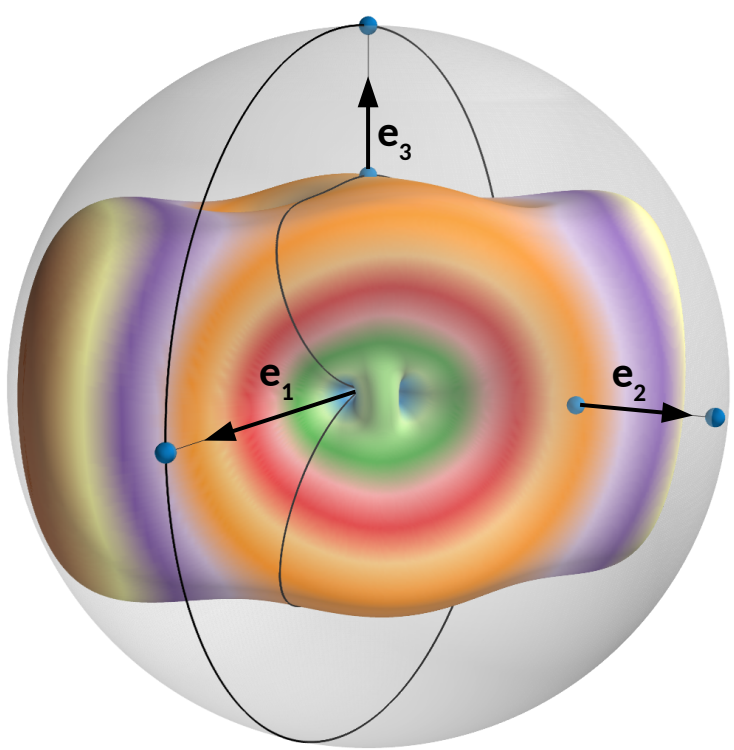

(b)

Figure 8: Polar plot of the elastic kernel with eigenstrain $\varepsilon_{\mathrm{T}_{3}}^{*}$ and isotropic elastic constants with (a) $A=1$ and (b) $A=5$. 2D computations are performed in plane $\left(\boldsymbol{e}_{3}, \boldsymbol{e}_{1}\right)$.

(3D) for all investigated supersaturations and eigenstrains in Fig. 12, plotting the tip radius with respect to $B_{\max }$. The collapse of all the results into a single curve for a given eigenstrain whatever the growth conditions suggests that the tip size would be an equilibrium property. Thus, we have computed equilibrium shapes for the simple eigenstrains, considering various box sizes, discretizations, and volume fractions (ranging from 3 to $11 \%$ to avoid any interactions with periodic images). To rapidly obtain equilibrium shapes, we have solved only AllenCahn equation (5) under the constraint of constant phase fraction. For a given $B_{\max }$, all calculations have converged toward the same equilibrium plate shape with a single tip radius (with variations smaller than fractions of $d$ ). In Fig. 12b for needles,

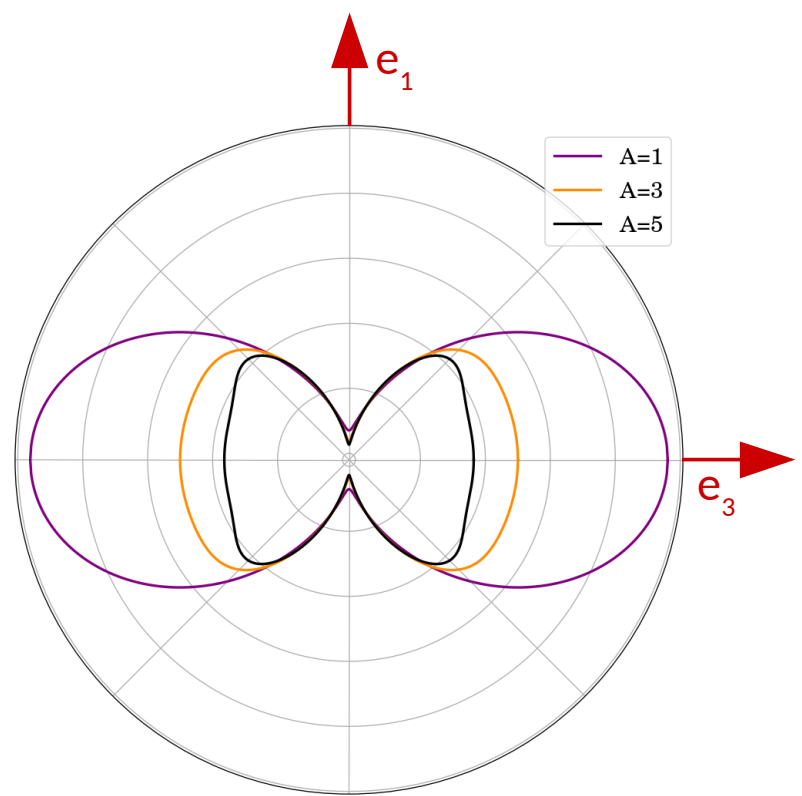

Figure 9: Polar plot of the elastic kernel for $\varepsilon_{\mathrm{Ti}}^{*}$ and two Zener factors $A=1$ (isotropic), $A=3$ and $A=5$.

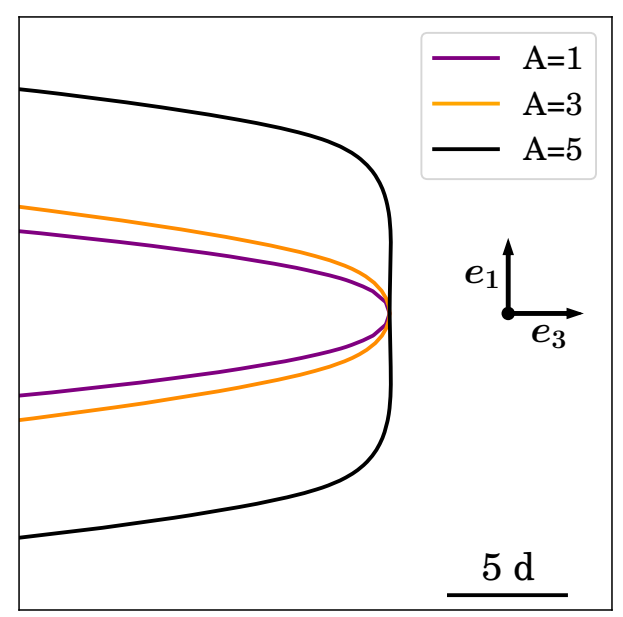

Figure 10: Tip shapes $(\varphi=0.5)$ during growth in the same conditions $\left(c_{\infty}=\right.$ 0.25 ) corresponding to Fig. 9 (the level sets have been shifted such that the tips are all at the same location)

the small discrepancies between the growth and equilibrium curves are smaller than the grid spacing $d$ and can be attributed to the interactions with periodic images due to smaller boxes than in $2 \mathrm{D}$. Using the analogy between plates and dislocations $[50,46,51]$, it is easy to understand that lengthening is energetically more favorable than thickening, such that equilibrium plate thickness increases only very slowly with length. Moreover, although the equilibrium plate thickness depends slightly on volume fraction, the tip radius is smaller than the plate thickness and do not exhibit any variations larger than a few percents of $d$, in the range of volume fractions considered. Unfortunately, we were not able to perform similar equilibrium cal- 


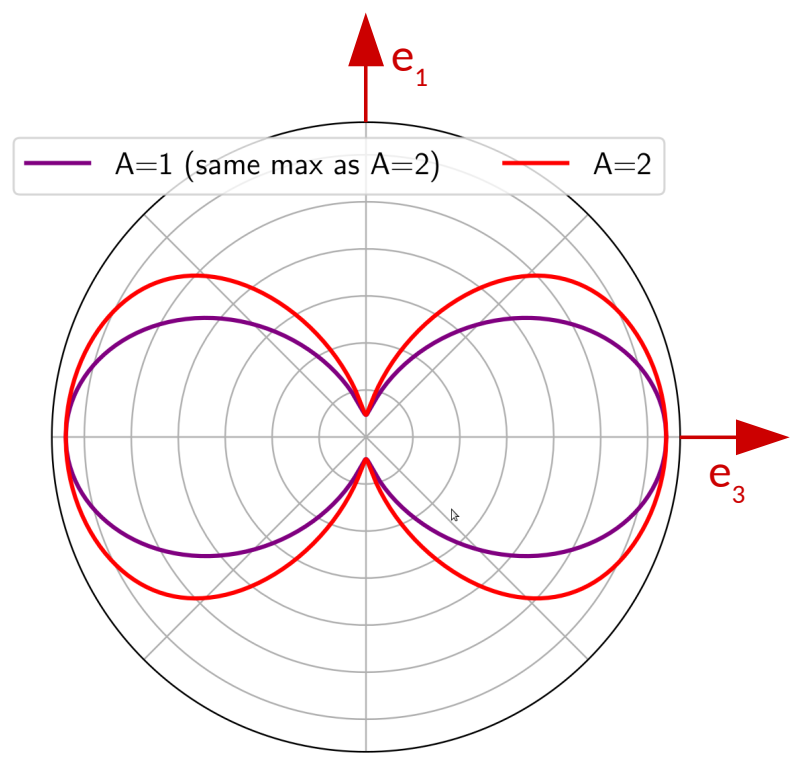

Figure 11: Polar plot of the elastic kernel for $\varepsilon_{\mathrm{Ti}}^{*}$ and two sets of elastic constants corresponding to $A=1$ and $A=2$ rescaled so as to display the same $B_{\max }$.

culations for eigenstrain (14) because of the significant elastic interactions between periodic images even in long boxes due to large $B_{\max }$. The resulting equilibrium values are plotted in Fig. 12 with black lines and white dots. The agreement between these equilibrium values and the steady growth values is excellent. We can conclude that the tip shape and size of Widmanstätten structures are equilibrium properties when only elasticity anisotropy is accounted for. It is likely to remain the main selecting phenomenon, even when other sources of anisotropy (interface energy and mobility) are present.

Using realistic scales for the parameters (\$2), typical values of the tip radius decreases from about $25 \mathrm{~nm}$ for $B_{\max }=$ $O\left(10^{-2}\right) \mathrm{GPa}$ to a few $\mathrm{nm}$ for $B_{\max }=O(1) \mathrm{GPa}$. The highest $B_{\max }$ are generated by eigenstrains with large shears, typically around $20 \%$ for $\varepsilon_{T i}^{*}$ corresponding to the red triangles in Fig. 12a. Tip radii are usually reported to span only one order of magnitude from tens to hundreds of nm (Tab. 1 and $[7,15,34,36,37])$. Although the selection curves in Fig. 12 are not universal as they depend on eigenstrains, elastic constants and interface energies, they still give clues about the elastic energy densities that must be conveyed at the tips to reach radii with experimental order of magnitudes. It is clear that $B_{\max }$ must be decreased by at least one order of magnitude for plates when considering eigenshear predicted by cristallographic analysis of transformations involving fcc, bcc and hep structures. It is worth stressing that increasing interfacial energy does not drastically change this statement because we had already considered a significant value of $\sigma_{i n t}=200 \mathrm{~mJ} \mathrm{~m}^{-2}$. Different phenomena can contribute to decrease the elastic energy at the tip. First, anisotropic elastic constants can have a significant impact (Fig. 10). Second, concentration fields can also play a role when the eigenstrain depends on concentration, e.g. following Vegard's law. Although preliminary calculations have shown that the relaxation associated with this process is mod-

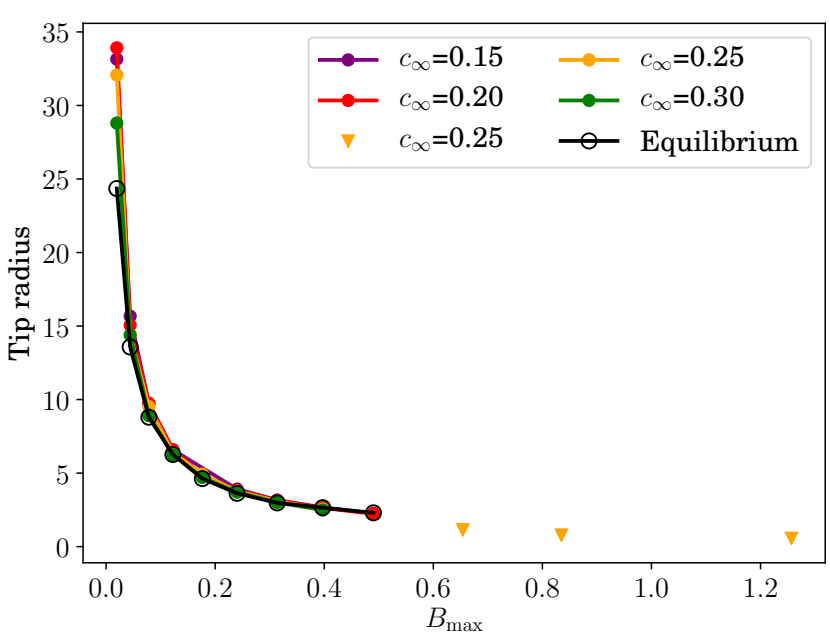

(a)

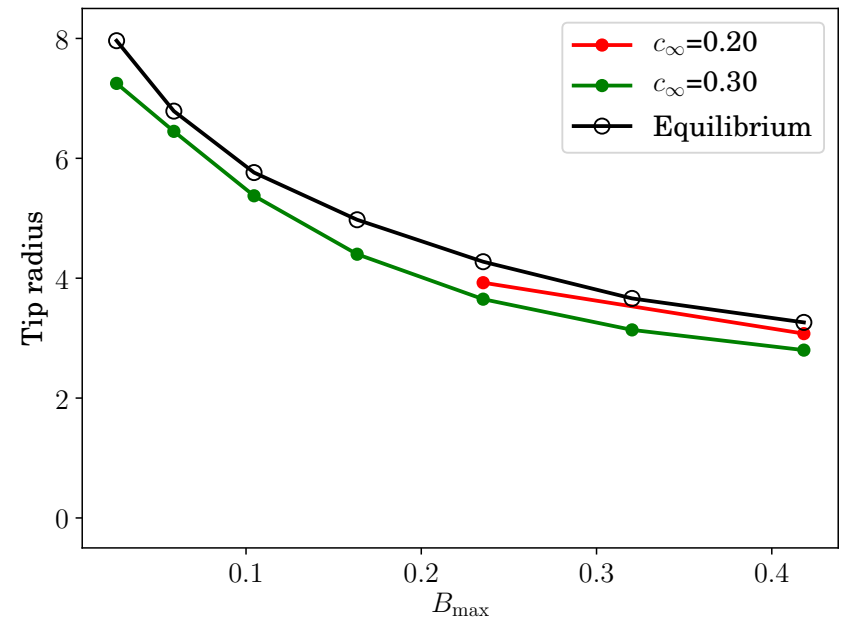

(b)

Figure 12: Tip radius vs. $B_{\max }$ for different supersaturations (colors) and different eigenstrains: (a) $\varepsilon_{2 \mathrm{D}}^{*}$ (circles) and $\varepsilon_{\mathrm{Ti}}^{*}$ (triangles) for plates (2D); (b) $\varepsilon_{3 D}^{*}$ for needles (3D). The corresponding equilibrium values are plotted with white symbols.

erate in the case of eigenstrains with perfect invariant planes [52], it could explain the slight variation of the tip radii measured by [35] at $700{ }^{\circ} \mathrm{C}$ with the carbon content (Tab. 1). Third, at high temperature, plasticity is likely to relax stresses at the tip. When described by isotropic $J_{2}$ constitutive laws, plasticity does not seem to change the selection process, keeping the same tip shape as shown in [53]. Finally, misfit compensating interfacial defects such as disconnections are likely to contribute significantly to relax elastic energy so that the tip radius increases. They can be accounted for by introducing an effective eigenstrain as shown in [42]. Hence, we expect that accounting for the relaxation processes will not change the conclusions of the present work about the selection of the tip shape, but will decrease $B_{\max }$ and increase the tip size in agreement with experimental observations. Still, below a sufficiently small elastic anisotropy, a crossover towards a dendrite-like growth with an- 
other selection criterion cannot be discarded.

A careful investigation of the effect of these relaxation processes on the tip selection is under progress.

\section{Conclusion}

Considering a broader range of supersaturations and eigenstrains than in [30], we have shown that the important features of Widmanstätten structures can be recovered by considering the anisotropic elastic energy generated by shear dominated eigenstrains: acicular morphologies (plates or needles) and constant lengthening rates at constant supersaturations. We have confirmed that the plates or needles lengthen at constant velocity along a particular direction perpendicular to two minima of the elastic kernel. We have also found that the Péclet number depends only on the supersaturation of the matrix and that this dependency is not far from Ivantsov analytical solutions for parabolic and paraboloidal shapes. One of the major outcome of the present study is that elasticity selects the tip shape and size, independently of the growth conditions. Consequently, when there is no other source of anisotropy than elasticity, the tip shape and size can be obtained by equilibrium calculations. We have shown numerically how the tip shape is related to the shape of the elastic kernel in the plane containing the habit plane normal and the lengthening direction. When the elastic kernel features in this plane a maximum in the lengthening direction, the tip is acicular. In this case, the tip size is shown to depend mainly on this maximum. Conversely, when, within this plane, the direction of the maximum of the elastic kernel deviates from the lengthening direction, the tip displays a blunted tip with a nearly flat side perpendicular to the lengthening direction.

The present work opens new perspectives for estimating the growth kinetics of Widmanstatten structures. First, our calculations have proved that Ivantsov solutions remain good estimates for the solute flow at the tips even when elasticity is playing a major role, changing the tip shape and the interface concentrations. Indeed, moderate differences with respect to these solutions have been found to be lower than common uncertainties on diffusion coefficients. This explains why the regular use of these solutions has been partly successful, e.g. [54] for a recent example. Second, we can deduce from the present study that the tip size can be computed independently from elasticity equilibrium calculations rather than ensuing from an ad hoc maximum velocity criterion where the interface energy is tuned. Moreover, our finding suggests to revisit the available experimental data and to perform new experiments where the difficult measurements of the tip sizes could be avoided, provided that the eigenstrain can be determined. Still, further work is needed to substantiate the new selection criterion, in particular how relaxation processes such as plasticity can be accounted for in equilibrium calculations.

\section{Appendix A. Elastic kernel for $\varepsilon_{2 D}^{*}$}

For isotropic elasticity, the Fourier transform of the Green function for displacements reads [46]:

$$
\Omega_{i j}(\boldsymbol{n})=\frac{\delta_{i j}}{\mu}-\frac{1}{2 \mu(1-v)} n_{i} n_{j}
$$

where $\mu$ is the shear modulus, and $v$ the Poisson ratio. For $\varepsilon_{2 D}^{*}$ Eq. (10), the eigenstress reads:

$$
\sigma_{i j}^{*}=-\varepsilon_{0}\left(\frac{2 \mu \nu}{1-2 \nu} \delta_{i j}-2 \mu \delta_{i 2} \delta_{j 2}\right)
$$

In the (001) plane we have $n_{3}=0$ such that $B(\boldsymbol{n})$ simplifies to:

$$
\begin{array}{r}
B(\boldsymbol{n})=\sigma_{22}^{*} \varepsilon_{0}-\left(\sigma_{11}^{*} n_{1}\right)^{2} \Omega_{11}-\left(\sigma_{22}^{*} n_{2}\right)^{2} \Omega_{22} \\
-2 \sigma_{11}^{*} n_{1} \sigma_{22}^{*} n_{2} \Omega_{12}
\end{array}
$$

with $n_{1}=\cos \theta$ and $n_{2}=\sin \theta$.

Using Eq. (A.2) and Eq. (A.1), after tedious but trivial algebra $B(\boldsymbol{n})$ becomes:

$$
B(\boldsymbol{n})=\frac{2 \mu}{1-v} \varepsilon_{0}^{2}(\cos \theta)^{4}
$$

\section{References}

\section{References}

[1] G. Thomson, On the malleable iron - essai sur le fer malléable trouvé en sibérie par le Prof. Pallas, Bibliothèque Britannique vol. 27 (1804) p. $135,154 \& 184$.

[2] F. Osmond, Microscopic Analysis of Metals, Charles Griffin \& Cie, 1904.

[3] R. Mehl, C. Barrett, Studies upon the Widmanstätten structure, i. - introduction. the aluminium-silver system and the copper-silicon system, AIME Transactions 93 (1930) 78-122.

[4] M. Belaiew, The Widmanstätten structure in various alloys and metals, Nature 94 (1914) 107-109.

[5] K. R. Kinsman, E. Eichen, H. I. Aaronson, Thickening kinetics of proeutectoid ferrite plates in Fe-C alloys, Metallurgical Transactions A 6 (1975) 303-317.

[6] C. Laird, H. I. Aaronson, The growth of $\gamma$-plates in an Al-15\% Ag alloy, Acta Metallurgica 17 (1969) 505-519.

[7] M. M. Kostic, E. B. Hawbolt, L. C. Brown, Growth kinetics of bainite plates and Widmanstätten needles in the $\beta$ phase of a Ag- 45 at. $\%$ cd alloy, Metallurgical Transactions A 7 (1976) 1643-1653.

[8] G. R. Purdy, Widmanstätten Precipitation from non-ideal solid solution: $\alpha$ in $\beta$-CuZn, Metal Science Journal 5 (1971) 81-85.

[9] D. Stephens, G. R. Purdy, On Widmanstätten growth from beta brass, Scripta Metallurgica 8 (1974) 323-328.

[10] E. K. Menon, H. Aaronson, Interfacial structure of widmanstätten plates in a ti-cr alloy, Acta Metallurgica 34 (1986) 1975-1981.

[11] B. Appolaire, L. Héricher, E. Aeby-Gautier, Modelling of phase transformation kinetics in ti alloys - isothermal treatments, Acta Materialia 53 (2005) 3001-3011.

[12] G. Ivantsov, Temperature field around a spherical, cylindrical, and needleshaped crystal, growing in a pre-cooled melt, Dokl. Akad. Nauk 58 (1947) 567-569.

[13] C. Zener, Theory of growth of spherical precipitates from solid solution, Journal of Applied Physics 20 (1949) 950-953.

[14] G. Horvay, J. Cahn, Dendritic and spheroidal growth, Acta Metallurgica 9 (1961) 695-705

[15] R. Trivedi, G. M. Pound, Growth kinetics of plate-like precipitates, Journal of Applied Physics 40 (1969) 4293-4300.

[16] M. Hillert, L. Höglund, J. Ågren, Diffusion-controlled lengthening of widmanstätten plates, Acta Materialia 51 (2003) 2089-2095. 
[17] H. Bhadeshia, Diffusion-controlled growth of ferrite plates in plaincarbon steels, Materials Science and Technology 1 (1985) 497-504.

[18] C. Atkinson, The growth kinetics of individual ledges during solid-solid phase transformations, Proceedings of the Royal Society A 378 (1981) 351-368.

[19] M. Enomoto, Computer modeling of the growth kinetics of ledged interphase boundaries - - i. single step and infinite train of steps, Acta Metallurgica 35 (1987) 935-945.

[20] M. Enomoto, Thermodynamics and kinetics of the formation of widmanstätten ferrite plates in ferrous alloys, Metallurgical and Materials Transactions A 25 (1994) 1947-1955.

[21] J. Hoyt, The velocity of plate precipitates growing by the ledge mechanism, Acta Materialia 61 (2013) 4953-4960.

[22] J. Dryden, L. Brown, G. Purdy, Elastic interactions of transformation ledges and precipitate edges, Scripta Metallurgica et Materialia 25 (1991) 2075-2078

[23] M. Enomoto, J. P. Hirth, Computer simulation of ledge migration under elastic interaction, Metallurgical and Materials Transactions A 27 (1996) 1491-1500.

[24] Y. Wang, A. G. Khachaturyan, Shape evolution of a precipitate during strain-induced coarsening - a computer simulation, Scripta Metallurgica et Materialia 25 (1991) 1387-1392.

[25] A. Finel, Y. Le Bouar, A. Gaubert, U. Salman, Phase field methods: Microstructures, mechanical properties and complexity, Comptes Rendus Physique 11 (2010) 245-256.

[26] K. Ammar, B. Appolaire, G. Cailletaud, S. Forest, Phase field modeling of elasto-plastic deformation induced by diffusion controlled growth of a misfitting spherical precipitate, Philosophical Magazine Letters 91 (2011) 164-172.

[27] M. Cottura, Y. Le Bouar, A. Finel, B. Appolaire, K. Ammar, S. Forest, A phase field model incorporating strain gradient viscoplasticity: Application to rafting in ni-base superalloys, Journal of the Mechanics and Physics of Solids 60 (2012) 1243-1256.

[28] L. Thuinet, A. De Backer, A. Legris, Phase-field modeling of precipitate evolution dynamics in elastically inhomogeneous low-symmetry systems: Application to hydride precipitation in zr, Acta Materialia 60 (2012) 5311-5321.

[29] V. de Rancourt, K. Ammar, B. Appolaire, S. Forest, Homogenization of viscoplastic constitutive laws within a phase field approach, Journal of the Mechanics and Physics of Solids 88 (2016) 291-319.

[30] M. Cottura, B. Appolaire, A. Finel, Y. Le Bouar, Phase field study of acicular growth: Role of elasticity in Widmanstätten structure, Acta Materialia 72 (2014) 200-210.

[31] B. Appolaire, A. Settefrati, E. Aeby-Gautier, Stress and strain fields associated with the formation of $\alpha$ " in near- $\beta$ titanium alloys, Materials Today: Proceedings 2 (2015) 589-592.

[32] A. Karma, Y. H. Lee, M. Plapp, Three-dimensional dendrite-tip morphology at low undercooling, Physical Review E 61 (2000) 3996-4006.

[33] B. Appolaire, E. Aeby-Gautier, M. Dehmas, A. Settefrati, M. Cottura, A. Finel, Y. Le Bouar, Modeling of phase transformations in $\beta$-metastable Ti alloys, in: V. Venkatesh, A. Pilchak, J. Allison, S. Ankem, R. Boyer, J. Christodoulou, H. Fraser, M. Ashraf Imam, Y. Kosaka, H. Rack, A. Chatterjee, A. Woodfield (Eds.), Ti-2015, the 13th World Conference on Titanium, TMS, Wiley Publishing, San Diego, USA, 2015, pp. 463 470.

[34] Z. Guo, W. Sha, Kinetics of ferrite to Widmanstätten austenite transformation in a high-strength low-alloy steel revisited, Zeitschrift für Metallkunde 95 (2004) 718-723.

[35] E. P. Simonen, H. I. Aaronson, R. Trivedi, Lengthening kinetics of ferrite and bainite sideplates, Metallurgical Transactions 4 (1973) 1239-1245.

[36] M. G. Hall, K. R. Kinsman, H. I. Aaronson, Mechanism of formation of $\mathrm{Mo}_{2} \mathrm{C}$ needles in an Fe-C-Mo alloy, Metallurgical and Materials Transactions B 3 (1972) 1320-1322.

[37] K. K. Sagoe-Crentsil, L. C. Brown, Dissolution kinetics of widmanstätten $\gamma-\mathrm{Ag}_{2} \mathrm{Al}$ precipitates, Metallurgical Transactions A 15 (1984) 19691975.

[38] W. B. Alexander, L. M. Slifkin, Diffusion of solutes in aluminium and dilute aluminium alloys, Physical Review B 1 (1970) 3274-3282.

[39] J. Ågren, A revised expression for the diffusivity of carbon in binary Fe-C austenite, Scripta Metallurgica 20 (1986) 1507-1510.

[40] A. Kuper, D. Lazarus, J. Manning, C. Tomizuka, Diffusion in ordered and disordered copper-zinc, Physical Review 104 (1956) 1536-1541.

[41] W. Burgers, On the process of transition of the cubic-body-centered modification into the hexagonal-close-packed modification of zirconium, Physica 1 (1934) 561-586.

[42] R. Shi, N. Ma, Y. Wang, Predicting equilibrium shape of precipitates as function of coherency state, Acta Materialia 60 (2012) 4172-4184.

[43] A. Settefrati, Etude expérimentale et modélisation par champ de phase de la formation de alpha dans les alliages de titane beta-métastable, Ph.D. thesis, Université de Lorraine, 2012.

[44] E. Kröner, Berechnung der elastischen konstanten des vielkristalls aus den konstanten des einkristalls, Zeitschrift Für Physik 151 (1958) 504518.

[45] H. Ledbetter, Handbook of Elastic Properties of Solids, Liquids, and Gases, volume III, Academic Press, San Diego, 2001, pp. 313-324.

[46] A. Khachaturyan, Theory of structural transformations in solids, John Wiley \& Sons, 1983.

[47] J. Langer, Instabilities and pattern formation in crystal growth, Reviews of Modern Physics 52 (1980) 1-28.

[48] A. Karma, Dendritic growth, in: V. Fleury, J. Gouyet, M. Léonetti (Eds.), Branching in Nature - Dynamics and morphogenesis of branching structures, from cell to river networks, Springer-Verlag, 1999, p. $365-401$.

[49] K. Kassner, E. Brener, Continuum description of noiseless diffusionlimited aggregation, Physical Review E 50 (1994) 2161-2165.

[50] F. Nabarro, Cxxii. the synthesis of elastic dislocation fields, The London, Edinburgh, and Dublin Philosophical Magazine and Journal of Science: Series 742 (1951) 1224-1231.

[51] D. Rodney, Y. Le Bouar, A. Finel, Phase field methods and dislocations, Acta Materialia 51 (2003) 17-30.

[52] H. Lebbad, Modélisation de la croissance des structures de Widmanstätten par la méthode des champs de phase, Ph.D. thesis, Sorbonne Université, 2018.

[53] M. Cottura, B. Appolaire, A. Finel, Y. Le Bouar, Plastic relaxation during diffusion-controlled growth of Widmanstätten plates, Scripta Materialia 108 (2015) 117-121.

[54] J. Yin, L. Leach, M. Hillert, A. Borgenstam, C-curves for lengthening of Widmanstätten and bainitic ferrite, Metallurgical and Materials Transactions A 48 (2017) 3997-4005. 